\title{
A novel missense variant of $L A M C 1$ as a possible cause of premature ovarian insufficiency in a Chinese family
}

\section{Huanfang Xu}

Acupuncture and Moxibustion Hospital of China Academy of Chinese Medical Sciences

\section{Chunyan Wang}

National Research Institute for Family Planning

Han Wei

National Research Institute for Family Planning

Tengyan Li

National Research Institute for Family Planning

\section{Yigong Fang}

Acupuncture and Moxibustion Hospital of China Academy of Chinese Medical Sciences

\section{Binbin Wang ( $\nabla$ wbbahu@163.com )}

National Research Institute for Family Planning

\section{Case Report}

Keywords: Premature ovarian insufficiency, Whole-exome sequencing, LAMC1, Missense variant, Autosomal dominant

Posted Date: February 23rd, 2022

DOI: https://doi.org/10.21203/rs.3.rs-1375481/v1

License: (우 This work is licensed under a Creative Commons Attribution 4.0 International License. Read Full License 


\section{Abstract}

Background: Premature ovarian insufficiency (POI) results in the loss of ovarian function and reproductive impairment. The cause of most cases of $\mathrm{POI}$ is unclear, although genetic factors are thought to be involved. This study aimed to identify novel pathogenic genes and variants in a Chinese family with POI.

Results: An assessment of the family pedigree suggested that POI was inherited in an autosomal dominant manner in this family. Whole-exome sequencing of the proband and her affected mother identified a novel heterozygous missense variant in the laminin subunit gamma-1 gene ( $L A M C 1$; NM_002293.4:c.A3281T:p.D1094V). This variant was not found in any public databases, and was highly conserved among mammals. Online software predicted it to be deleterious with respect to protein function. Its presence in the POI family was confirmed by Sanger sequencing.

Conclusions: We report a novel heterozygous missense variant in $L A M C 1$ in a Chinese POI family, which was inherited in an autosomal dominant manner. This variant may result in the development of POI. Our results provide supporting evidence for a causative role for $\angle A M C 1$ variants in $\mathrm{POI}$.

\section{Background}

Premature ovarian insufficiency $(\mathrm{POI})$ is a serious reproductive disorder characterized by the depletion or loss of normal ovarian function in women under 40 years of age. It affects about $1 \%$ of women of childbearing age worldwide, and typically manifests with $\mathrm{a} \geq 4$-month history of oligomenorrhea/amenorrhea and follicle-stimulating hormone (FSH) levels $>25 \mathrm{IU} / \mathrm{L}$ in two measurements for at least 4 weeks [1, 2]. Hormone replacement therapy (HRT) can partially alleviate the symptoms caused by POI, but there are limited effective treatments for the reproductive impairment [3].

The etiologies of $\mathrm{POI}$ are complex, and mainly including genetic, environmental, autoimmune, or other factors [4]; however, the causes of most POI cases remain unclear.

Several studies have suggested that genetic factors play an important role in POI [5], and various genes have been identified to harbor variants that affect the biological function associated with POI. With the rapid development of sequencing technology, whole-exome sequencing (WES) is now widely used, and has proven useful in POI gene discovery [6]. Multiple genes associated with POI have been identified in affected families using WES, including BRCA2, PSMC3IP, SYCP2L, BUB1B, STAG3, SPIDR, POLR2C, MEIOB, MCM8, MCM9, MRPS22, FIGLA, and PMM2 [7-17]. Similarly, WES has identified genes associated with POI in sporadic patients, including NANOS3, BNC1, EIF2B4, FOXL2, FANCA, SALL4, EIF2B3, and GHR [6, 18-21].

Considering that $\mathrm{POI}$ is a highly heterogeneous disease, a better understanding of the underlying mechanisms and genetic etiology is needed. Additionally, very few families with autosomal dominant POI have been described and assessed by WES to identify causative genes. Here, we performed WES in a Chinese family with $\mathrm{POI}$ and identified a novel rare heterozygous missense variant in the laminin subunit gamma-1 gene (LAMC1). Our findings expand the spectrum of POI causative variants, which may be useful for future pathogenic studies. 


\section{Results}

\section{Clinical findings}

A Chinese family including two POI patients was enrolled in this study (Fig. 1). The 29-year-old proband (II1) had undergone normal puberty, and menarche at 12 years of age. She had attended our hospital for the treatment of menopause for more than 3 months. Prior to this, she had experienced irregular menstruation more than one year, but no dysmenorrhea, headache, or other discomfort. There was no history of ovarian surgery, chemotherapy, radiotherapy, or immune disease.

Physical examination showed a normal body mass index. FSH levels on two separate occasions $>4$ weeks apart were 29.15 and $25.62 \mathrm{IU} / \mathrm{L}$, respectively (Table 1 ). Antral follicle counting on the third day of menstruation revealed no follicles in either ovary. Ultrasound examination showed the uterus to be $4.0 \times 4.2$ $\times 3.6 \mathrm{~cm}$, an endometrial thickness of $0.35 \mathrm{~cm}$, and right and left ovary sizes of $2.6 \times 0.9 \times 1.2 \mathrm{~cm}$ and $2.2 \times$ $1.2 \times 0.9 \mathrm{~cm}$, respectively. There was no obvious follicular echo in either ovary.

The proband's mother started to have irregular menstrual cycles at the age of 34 years, and received HRT at the age of 38 years because of amenorrhea that had persisted for more than 3 months.

\section{Identification of the missense LAMC1 variant}

To investigate the genetic cause of POI in this family, we performed WES on the patient and her mother. Unrelated mutations were excluded from sequencing results by rigorous bioinformatics analysis. In silico prediction revealed 50 deleterious variants, including six non-frameshift deletion variants, one frameshift deletion variant, two splicing variants, four nonsense variants, and 37 missense variants (Additional file 1).

One of the novel missense variants was in $\angle A M C 1$ (c.A3281T: p.D1094V), which was previously identified as a gene associated with $\mathrm{POI}$ [22]. Sanger sequencing confirmed that the proband and her mother carried the same missense variant (Fig. 2a).

\section{In silico analysis and alignment of the missense LAMC1 variant}

The missense LAMC1 variant was shown to be located within domains I and II of the protein (Fig. 2b). The minor allele frequency (MAF) of the variant was assessed in East Asian and global populations using the GnomAD database. Next, the impact of the variant on protein function was predicted by various bioinformatic tools. As shown on Table 3, The variant is absent from both East Asian and global populations. It was shown to be damaging by Sorting Intolerant From Tolerant (SIFT), possibly damaging by Polymorphism Phenotyping v2 (PolyPhen-2), and disease-causing by MutationTaster. Its Combined Annotation-Dependent Depletion (CADD) score was 26.5, and its American College of Medical Genetics (ACMG) classification was a variant of unknown significance (PM2, PP3).

Furthermore, the LAMC1 variant was found to be strongly conserved among species (Fig. 2c). Above results suggested a high pathogenicity of this variant and we hypothesized that this heterozygous missense variant of $\angle A M C 1$ might be the cause of this POI family. 


\section{Discussion}

In this study, we investigated the genetic cause of POI in a Chinese family with two affected females. The filtering of variants obtained by WES identified a novel variant of $\angle A M C 1$, which was potentially causative of $\mathrm{POI}$. Our study further provides evidence that $\angle A M C 1$ variants are important in the pathogenesis of POI.

Laminins are a family of extracellular matrix proteins that are associated with the development of ovarian follicles $[23,24]$. They play an important role in cell adhesion, differentiation, migration, and signaling through interactions with integrins, dystroglycan, and other proteins [25]. Mature laminins are crucial components of the basal lamina; together with collagens they affect the structure and function of numerous tissues [26].

Laminins consist of three identical chains: laminin alpha, beta, and gamma (formerly A, B1, and B2, respectively). Each laminin chain is a multidomain protein encoded by a distinct gene which shows tissuespecific expression that changes during differentiation, resulting in the expression of specific mature laminin tripeptides in different tissues and cell types. Previous immunostaining studies have shown that some laminin chains, including the $\gamma 1$ chain, are expressed in the basal lamina, and granulosa and/or theca cells of ovarian follicles in various animals [27,28], and in the human basal lamina around blood vessels and stroma, including theca interna cells of preovulatory follicles [29]. Increased expression of laminins was documented in the bovine basal lamina during follicular development [30], and similarly observed in pig and rabbit models [28]. Laminins also affect the proliferation, survival, and steroidogenesis of human granulosa cells (GCs) in vitro, suggesting that they play a role in folliculogenesis [31].

$\angle A M C 1$ encodes the $\gamma 1$ chain, which is the most abundantly expressed laminin subunit. $L A M C 1$ is located on chromosome 1q25.3, contains 28 exons, and encodes a protein of 1609 amino acids that is expressed in a variety of human tissues, including the placenta, lung, heart, breast, and ovaries. According to the STRING database, LAMC1 might interacts with other laminin subunits such as LAMA3, LAMA5, and LAMB2 to affect laminin protein structure and function .

Previous human studies reported that $L A M C 1$ was mainly expressed in follicular GCs, and that laminin tripeptides containing $Y 1$ increased follicular survival in vitro [32]. LAMC1 overexpression was found to enhance tumor cell invasion and migration and to predict poor ovarian cancer prognosis [33], while LAMC1 polymorphisms were associated with an increased risk of developing pelvic organ prolapse and $\mathrm{POI}[22$, 34]. Furthermore, absence of the laminin $Y 1$ chain by $L A M C 1$ deletion in mouse models led to aberrant basal lamina formation and embryonic lethality through the prevention of entire laminin polymerization [35]. Laminin-null Schwann cells also exhibited the depletion of all other laminin chains, together with reduced phosphatidylinositol 3 kinase activity and activation of caspase cascades, leading to cell apoptosis $[36,37]$. We speculate that the disruption of $\angle A M C 1$ causes $\mathrm{POI}$ by similarly preventing polymerization of the entire laminin molecule, resulting in the abnormal regulation of GC survival, which has a major role in follicular atresia.

In a recent genome-wide association study, Pyun et al. [22] found that 22 single nucleotide polymorphisms (SNPs) of $L A M C 1$ formed a linkage disequilibrium block in $\mathrm{POI}$ patients. This study showed that 
possession of at least one LAMC1 haplotype (C-C-T-G-C-C-A-T-T-C) and nine LAMC1 SNPs were associated with susceptibility to $\mathrm{POI}$ in the dominant model, suggesting that $\angle A M C 1$ is involved in POI pathogenesis. This genetic model is consistent with that of the family in our study. However, there have been no previous reports about rare and potentially pathogenic variants of $L A M C 1$ in POI patients, and especially in familial POI cases. Here, we used WES to identify a novel heterozygous missense variant c.A3281T (p.D1094V) in $\angle A M C 1$ in the proband and her affected mother of a Chinese POI family. The mutated site is located in exon 19 of $L A M C 1$, within domains I and II of the protein which are thought to interact with other laminin chains to form a coiled-coil structure according to the uniprot database. Furthermore, it is highly conserved among mammals, and the variant was predicted to be deleterious in in silico analysis. However, its effect on laminin protein function should be investigated in functional studies.

\section{Conclusions}

In summary, the present study identified a novel heterozygous missense variant in $L A M C 1$ (NM_002293.4:c.A3281T:p.D1094V) in a Chinese family with POI, which was inherited in an autosomal dominant manner. We speculate that this variant is causative of POI, and our findings suggest the importance of WES in the early detection and intervention of POI. Further studies are required to clarify the association between the variant and POI through functional experiments, and larger sample sizes are needed to explore other genetic causes of POI.

\section{Materials And Methods}

\section{Study subjects}

A Chinese family with POI was enrolled in this study from the Acupuncture and Moxibustion Hospital of the China Academy of Chinese Medical Sciences. The proband was diagnosed with POI according to previously described criteria [38]: oligomenorrhea/amenorrhea for at least 4 months, and an elevated FSH level $>25$ IU/L on two occasions $>4$ weeks apart. Her mother started irregular menstrual cycles at 34 years of age and developed amenorrhea at the age of 40 years. Both patients have a normal 46,XX karyotype. Five ml of peripheral blood was collected from the proband and her parents. This study was in accordance with the principles of the Declaration of Helsinki, and was approved by the ethics committee of the Institute of Acupuncture and Moxibustion, China Academy of Chinese Medical Sciences. Written informed consent was obtained from all participants.

\section{DNA extraction}

Genomic DNA was extracted from peripheral blood using a QIAamp DNA Blood Mini Kit (Qiagen, Hilden, Germany) according to the manufacturer's instructions. The quality of the DNA samples was assessed using a NanoDrop2000 spectrophotometer (Thermo Fisher Scientific, Waltham, MA).

\section{WES analysis}


WES was performed on the proband and her mother. The patients' exomes were captured using a SureSelect Human All Exon V6 Enrichment kit (Agilent, Santa Clara, CA), and then sequenced on a NovaSeq platform (Illumina, San Diego, CA) according to the manufacturer's guidance. All reads were mapped to the human reference genome (hg19) using Burrows-Wheeler Alignment version 0.7.9a (http://biobwa.sourceforge.net). Single nucleotide variants and indels were detected using Genome Analysis Toolkit version 3.5 software (https://gatk.broadinstitute.org/hc/en-us) and annotated using ANNOVAR (https://annovar.openbioinformatics.org/en/latest/user-guide/download/) by consideration of splice site, intronic, exonic, 5' untranslated region (UTR), 3' UTR, intergenic, upstream, or downstream locations.

\section{Variant filtering}

Heterozygous variants shared by the proband and her affected mother were selected according to the following criteria: i) missense, nonsense, frameshift, non-frameshift, or splicing site variants; ii) variants with a MAF $<0.1 \%$ in East Asians and the total population in the 1000 Genomes Project (1KG Project; http://browser.1000genomes.org) and GnomAD (https://gnomad.broadinstitute.org) dataset. Predictions of deleterious nonsynonymous variants were performed using four following online software programs: SIFT (http://sift-dna.org), PolyPhen-2 (http://genetics.bwh.harvard.edu/pph2/), MutationTaster (http://www.mutationtaster.org), and CADD (http://cadd.gs.washington.edu), which integrates information from various functional annotations and presents this as a score. CADD pathogenicity prediction scoring > 20 predicts the top $1 \%$ of deleterious variants [39]. The final candidate variant was evaluated according to ACMG variant interpretation guidelines.

\section{Sanger sequencing validation}

The final candidate variant was validated in family members using standard Sanger sequencing. Primer5 software was used to design specific primers which are shown in Table 2.

\section{Alignment of the LAMC1 protein}

Alignment of the LAMC1 protein among different species (human, mouse, rat, cattle, wild pig, Xenopus, and medaka) was performed using CLC Sequence Viewer 8 software to investigate sequence conservation.

\section{Abbreviations}

POI: Premature ovarian insufficiency; WES: Whole-exome sequencing; HRT: Hormone replacement therapy; FSH: Follicle-stimulating hormone; LAMC1: Laminin subunit gamma-1; SNPs: Single nucleotide polymorphisms; ACMG: American College of Medical Genetics and Genomics; GCs: Granulosa cells; CADD: Combined Annotation-Dependent Depletion; SIFT: Sorts Intolerant from Tolerant; MAF: Minor allele frequency; UTR: Untranslated region.

\section{Declarations}

\section{Ethics approval and consent to participate}


The study was approved by the ethics committee of the Institute of Acupuncture and Moxibustion, China Academy of Chinese Medical Sciences. Written informed consent was obtained from all participants.

\section{Consent for publication}

All authors read and approved the final manuscript.

\section{Availability of data and materials}

All data used during the study are available from the corresponding author on reasonable request.

\section{Competing interests}

The authors declare that they have no competing interests.

\section{Funding}

This study was supported by the National Natural Science Foundation of China (No. 81904308) and CAMS Innovation Fund for Medical Sciences (2018-I2M-1-004).

\section{Authors' contributions}

All authors contributed to the study conception and design. HX and YF collected the clinical samples and performed the clinical diagnosis. BW, CW and HW analysed WES data. The validation experiment was performed by TL; The first draft of the manuscript was written by $\mathrm{CW}$ and HX. All authors commented on early versions of the manuscript. All authors read and approved the final manuscript.

\section{Acknowledgements}

We would like to acknowledge all study participants.

\section{References}

1. Chon SJ, Umair Z, Yoon MS. Premature Ovarian Insufficiency: Past, Present, and Future. Frontiers in cell and developmental biology 2021; 9:672890.

2. McGlacken-Byrne SM, Conway GS. Premature ovarian insufficiency. Best practice \& research. Clinical obstetrics \& gynaecology 2021.

3. Huang QY, Chen SR, Chen JM, Shi QY, Lin S. Therapeutic options for premature ovarian insufficiency: an updated review. Reproductive biology and endocrinology: RB\&E 2022; 20(1):28.

4. Ishizuka B. Current Understanding of the Etiology, Symptomatology, and Treatment Options in Premature Ovarian Insufficiency (POI). Frontiers in endocrinology 2021; 12:626924.

5. Jiao X, Ke H, Qin Y, Chen ZJ. Molecular Genetics of Premature Ovarian Insufficiency. Trends in endocrinology and metabolism: TEM 2018; 29(11):795-807. 
6. Liu H, Wei X, Sha Y, Liu W, Gao H, Lin J, et al. Whole-exome sequencing in patients with premature ovarian insufficiency: early detection and early intervention. Journal of ovarian research 2020; 13(1):114.

7. He WB, Tan C, Zhang YX, Meng LL, Gong F, Lu GX, et al. Homozygous variants in SYCP2L cause premature ovarian insufficiency. Journal of medical genetics 2021; 58(3):168-72.

8. Chen B, Li L, Wang J, Li T, Pan H, Liu B, et al. Consanguineous familial study revealed biallelic FIGLA mutation associated with premature ovarian insufficiency. Journal of ovarian research 2018; $11(1): 48$.

9. Zhang YX, He WB, Xiao WJ, Meng LL, Tan C, Du J, et al. Novel loss-of-function mutation in MCM8 causes premature ovarian insufficiency. Molecular genetics \& genomic medicine 2020; 8(4):e1165.

10. Chen Q, Ke H, Luo X, Wang L, Wu Y, Tang S, et al. Rare deleterious BUB1B variants induce premature ovarian insufficiency and early menopause. Human molecular genetics 2020; 29(16):2698-707.

11. Jaillard S, McElreavy K, Robevska G, Akloul L, Ghieh F, Sreenivasan R, et al. STAG3 homozygous missense variant causes primary ovarian insufficiency and male non-obstructive azoospermia. Molecular human reproduction 2020; 26(9):665-77.

12. Heddar A, Guichoux N, Auger N, Misrahi M. A SPIDR homozygous nonsense pathogenic variant in isolated primary ovarian insufficiency with chromosomal instability. Clinical genetics 2022; 101(2):242-6.

13. Moriwaki M, Moore B, Mosbruger T, Neklason DW, Yandell M, Jorde LB, et al. POLR2C Mutations Are Associated With Primary Ovarian Insufficiency in Women. Journal of the Endocrine Society 2017; 1(3):162-73.

14. Peng T, Lv C, Tan H, Huang J, He H, Wang Y, et al. Novel PMM2 missense mutation in a Chinese family with non-syndromic premature ovarian insufficiency. Journal of assisted reproduction and genetics 2020; 37(2):443-50.

15. Caburet S, Todeschini AL, Petrillo C, Martini E, Farran ND, Legois B, et al. A truncating MEIOB mutation responsible for familial primary ovarian insufficiency abolishes its interaction with its partner SPATA22 and their recruitment to DNA double-strand breaks. EBioMedicine 2019; 42:524-31.

16. Chen A, Tiosano D, Guran T, Baris HN, Bayram Y, Mory A, et al. Mutations in the mitochondrial ribosomal protein MRPS22 lead to primary ovarian insufficiency. Human molecular genetics 2018; 27(11):1913-26.

17. Anon. !!! INVALID CITATION !!!

18. Sousa BL, Nishi MY, Santos MG, Brito VN, Domenice S, Mendonca BB. Mutation analysis of NANOS3 in Brazilian women with primary ovarian failure. Clinics 2016; 71(12):695-8.

19. Yang X, Touraine P, Desai S, Humphreys G, Jiang H, Yatsenko A, et al. Gene variants identified by wholeexome sequencing in 33 French women with premature ovarian insufficiency. Journal of assisted reproduction and genetics $2019 ; 36(1): 39-45$.

20. Wang Q, Li D, Cai B, Chen Q, Li C, Wu Y, et al. Whole-exome sequencing reveals SALL4 variants in premature ovarian insufficiency: an update on genotype-phenotype correlations. Human genetics 2019; 138(1):83-92. 
21. Grzechocinska B, Warzecha D, Wypchlo M, Ploski R, Wielgos M. Premature ovarian insufficiency as a variable feature of blepharophimosis, ptosis, and epicanthus inversus syndrome associated with c.223C > T p.(Leu75Phe) FOXL2 mutation: a case report. BMC medical genetics 2019; 20(1):132.

22. Pyun JA, Cha DH, Kwack K. LAMC1 gene is associated with premature ovarian failure. Maturitas 2012; 71(4):402-6.

23. Irving-Rodgers HF, Rodgers RJ. Extracellular matrix of the developing ovarian follicle. Seminars in reproductive medicine 2006; 24(4):195-203.

24. Irving-Rodgers HF, Rodgers RJ. Extracellular matrix in ovarian follicular development and disease. Cell and tissue research 2005; 322(1):89-98.

25. Durbeej M. Laminins. Cell and tissue research 2010; 339(1):259-68.

26. Domogatskaya A, Rodin S, Tryggvason K. Functional diversity of laminins. Annual review of cell and developmental biology 2012; 28:523-53.

27. Leardkamolkarn V, Abrahamson DR. Immunoelectron microscopic localization of laminin in rat ovarian follicles. The Anatomical record 1992; 233(1):41-52.

28. Lee VH, Britt JH, Dunbar BS. Localization of laminin proteins during early follicular development in pig and rabbit ovaries. Journal of reproduction and fertility 1996; 108(1):115-22.

29. Fujiwara H, Honda T, Ueda M, Nakamura K, Yamada S, Maeda M, et al. Laminin suppresses progesterone production by human luteinizing granulosa cells via interaction with integrin alpha 6 beta 1. The Journal of clinical endocrinology and metabolism 1997; 82(7):2122-8.

30. Zhao Y, Luck MR. Gene expression and protein distribution of collagen, fibronectin and laminin in bovine follicles and corpora lutea. Journal of reproduction and fertility 1995; 104(1):115-23.

31. Berkholtz CB, Shea LD, Woodruff TK. Extracellular matrix functions in follicle maturation. Seminars in reproductive medicine 2006; 24(4):262-9.

32. Hao J, Tuck AR, Prakash CR, Damdimopoulos A, Sjodin MOD, Lindberg J, et al. Culture of human ovarian tissue in xeno-free conditions using laminin components of the human ovarian extracellular matrix. Journal of assisted reproduction and genetics 2020; 37(9):2137-50.

33. Diao B, Yang P. Comprehensive Analysis of the Expression and Prognosis for Laminin Genes in Ovarian Cancer. Pathology oncology research: POR 2021; 27:1609855.

34. Chen J, Li L, Lang J, Zhu L. Common variants in LAMC1 confer risk for pelvic organ prolapse in Chinese population. Hereditas 2020; 157(1):26.

35. Smyth N, Vatansever HS, Murray P, Meyer M, Frie C, Paulsson M, et al. Absence of basement membranes after targeting the LAMC1 gene results in embryonic lethality due to failure of endoderm differentiation. The Journal of cell biology 1999; 144(1):151-60.

36. Yu WM, Feltri ML, Wrabetz L, Strickland S, Chen ZL. Schwann cell-specific ablation of laminin gamma1 causes apoptosis and prevents proliferation. The Journal of neuroscience: the official journal of the Society for Neuroscience 2005; 25(18):4463-72.

37. McKee KK, Yang DH, Patel R, Chen ZL, Strickland S, Takagi J, et al. Schwann cell myelination requires integration of laminin activities. Journal of cell science 2012; 125(Pt 19):4609-19. 
38. European Society for Human R, Embryology Guideline Group on POI, Webber L, Davies M, Anderson R, Bartlett J, et al. ESHRE Guideline: management of women with premature ovarian insufficiency. Human reproduction 2016; 31(5):926-37.

39. Kircher M, Witten DM, Jain P, O'Roak BJ, Cooper GM, Shendure J. A general framework for estimating the relative pathogenicity of human genetic variants. Nature genetics $2014 ; 46(3): 310-5$.

\section{Tables}

Table 1 Hormonal characteristics of the proband

\begin{tabular}{|cccll|}
\hline NO. & Period & $\begin{array}{l}\text { FSH } \\
(\mathrm{IU} / \mathrm{L})\end{array}$ & $\begin{array}{l}\text { LH } \\
(\mathrm{IU} / \mathrm{L})\end{array}$ & $\begin{array}{l}\text { E2 } \\
(\mathrm{pmol})\end{array}$ \\
\hline 1 & $\mathrm{M} 3$ & 29.15 & 8.33 & 108 \\
\hline 2 & $\mathrm{M} 3$ & 25.62 & 8.02 & 160 \\
\hline
\end{tabular}

Abbreviation: M3, the third day of menstruation; FSH, Follicle-stimulating hormone; LH, Luteinizing hormone; E2, Estradiol

Table 2 LAMC1-specific primers used for Sanger sequencing

\begin{tabular}{|lll|}
\hline ID & Sequence & Length (bp) \\
\hline LAMC1- F & ACATTCCTTGGGTGTCTT & 459 \\
\hline LAMC1- R & TTTCAATCAACCGCTCTG & \\
\hline
\end{tabular}

Table 3 Biological analysis of the LAMC1 missense variant c.A3281T

\begin{tabular}{|c|c|c|c|c|c|c|c|c|}
\hline \multirow{2}{*}{$\begin{array}{l}\text { Chromosome } \\
\text { Position }\end{array}$} & \multirow{2}{*}{$\begin{array}{l}\text { Gene } \\
\text { Variant }\end{array}$} & \multirow{2}{*}{$\begin{array}{l}\text { Amino } \\
\text { acid } \\
\text { change }\end{array}$} & \multirow[t]{2}{*}{ Frequency\$ } & \multicolumn{4}{|c|}{ Online Prediction } & \multirow{2}{*}{$\begin{array}{l}\text { ACMG } \\
\text { Classification }\end{array}$} \\
\hline & & & & SIFT & PP2 & MT & CADD & \\
\hline chr1: & c.A3281T & p.D1094V & absent & D & $P$ & D & 26.5 & AUS \\
\hline 183099479 & & & & & & & & (PM2,PP3) \\
\hline
\end{tabular}

\$Frequency in overall population/East Asian population in gnomAD.

Abbreviation: SIFT, Sorts Intolerant from Tolerant (D, damaging); PP2, Polyphen-2 (P, possibly damaging); MT, MutationTaster (D, disease-causing); CADD, Combined Annotation-Dependent Depetion.

\section{Figures}




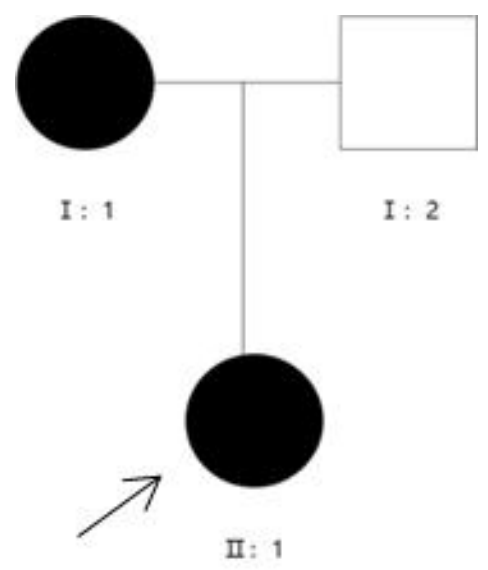

\section{Figure 1}

Pedigree of the family with POI. Filled black symbols represent affected members. Arrow denotes the proband. 
A

C CATTTTAGATGTTGACCA

I-1

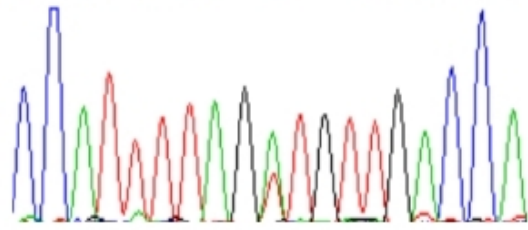

I-2

C C A T T TA A T T T A A C A

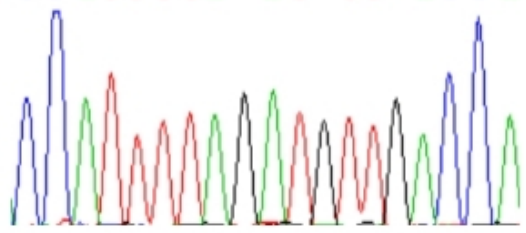

II-1

C CA T T T TA G A T TTG A C C A

B
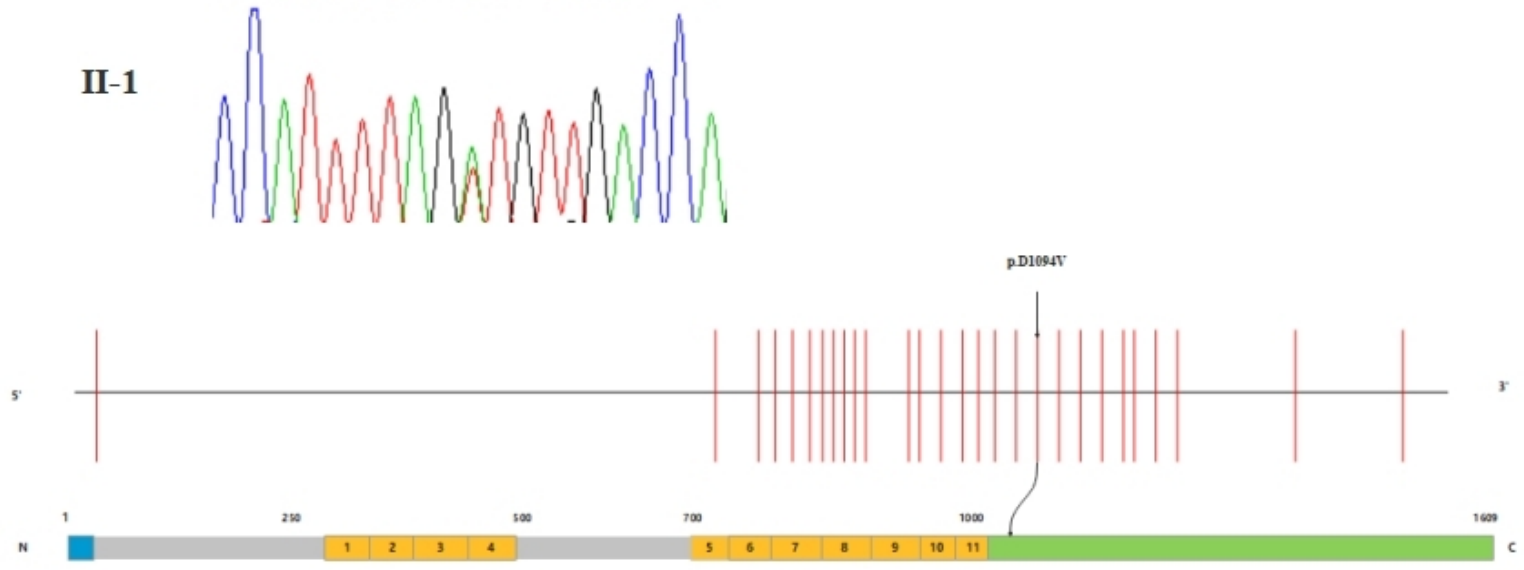

signal peptise $\quad$ EGF-like Domin I Ind II

C

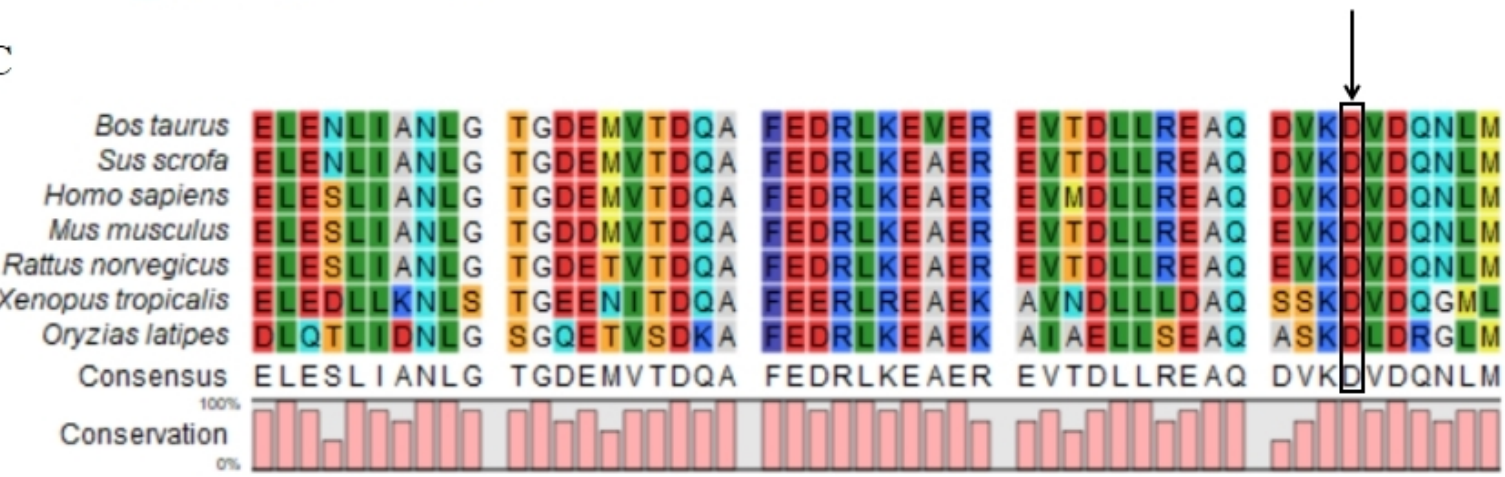

\section{Figure 2}

Genetic and protein analysis of the LAMC1 missense variant. a The chromatogram of the missense variant c.A3281T (p.D1094V) of LAMC1 in the patient and his parents. The arrow denotes the mutation site. $\mathbf{b}$ The location of the mutation in the intron-exon structure of LAMC1 and the protein domain map of LAMC1. The missense variant c.A3281T (p.D1094V) of LAMC1 is located in the domain $\nabla$ and $\nabla$. c Amino acid alignment of the LAMC1 protein from several organisms. The position of Asp1094 residue (highlighted by a black box) was highly conserved among different species 


\section{Supplementary Files}

This is a list of supplementary files associated with this preprint. Click to download.

- supplementarytable.docx 